\title{
Filtering Techniques to reduce Speckle Noise and Image Quality Enhancement methods on Satellite Images
}

\author{
K.M. Sharavana Raju ${ }^{1}$, Mohammad Shahnawaz Nasir ${ }^{2}$, T. Meera Devi ${ }^{3}$ \\ Lecturer, Department of Computer Science, Jazan University, Jazan, Kingdom of Saudi Arabial ${ }^{l}$ \\ Lecturer, Department of Computer Science, Jazan University, Jazan, Kingdom of Saudi Arabia ${ }^{2}$ \\ Associate Professor, Department of ECE, Kongu Engg College, Perundurai, Tamilnadu, India ${ }^{3}$
}

\begin{abstract}
Noise in images is the vital factor which degrades the quality of the images. Reducing noise from the satellite images, medical images etc., is a challenge for the researchers in digital image processing. Several approaches are there for noise reduction. Generally speckle noise is commonly found in Synthetic Aperture Radar (SAR) satellite images and medical images. This research paper put forward some of the filtering techniques for the removal of speckle noise from the satellite images, which enhances the quality of the images. Although many filters are available for speckle reduction, some filters are best suited for SAR images are used for which the statistical parameters are calculated for the output images obtained from all the filters. The statistical measures SNR, PSNR, RMSE and CoC are compared. The output images corresponding to the best statistical values are displayed along with the filters name and corresponding values of the statistical measures.

Keywords: Filters, Speckle noise reduction, Image enhancement, Satellite images, Statistical measures.
\end{abstract}

\section{Introduction}

Satellite images are usually degraded by noise during image acquisition and transmission process. The main purpose of the noise reduction technique is to remove speckle noise by retaining the important feature of the images. Mathematically there are two basic models of noise: Additive noise and Multiplicative noise. Additive noise is systematic in nature and can be easily modeled and hence removed or reduced easily; whereas multiplicative noise is image dependent, complex to model and hence difficult to reduce. Speckle [1] is not a noise in an image but noise-like variation in contrast. Speckle is mainly a form of multiplicative noise, which occurs when a sound wave pulse arbitrarily interferes with the small particles or objects on a scale comparable to the sound wavelength. Speckle noise is defined as multiplicative noise, having a granular pattern it is the inherent property of SAR image.

Synthetic Aperture Radar (SAR) imagery uses microwave radiation so that it can illuminate the earth surface. Synthetic Aperture Radar provides its own illumination. It is not affected by cloud cover or radiation in solar illumination. ISUKF technique [2] which uses sampling to incorporate the Discontinuity - adaptive Markov random field for the reduction of speckle noise. Context-based adaptive wavelet thresholding [3] method introduced a simple context-based method for the selection of adaptive threshold. Coherent filtering [4] is a speckle noise reduction technique of the SAR images. This technique is based on Coherent Anisotropic Diffusion for real time adaptive Speckle noise reduction.

\subsection{Major reasons of speckle noise}

Due to incorrect assumptions that the ultrasound pulse always travel in a straight line, to and fro from the reflecting interference. Another source of reverberations is that a small portion of the returning sound pulse may be reflected back into the tissues by the transducer surface itself, and generates a new echo at twice the depth. Speckle is the result of the diffuse scattering, which takes place when an ultrasound pulse randomly interferes with the small particles or objects on a scale comparable to the sound wavelength. The backscattered echoes from irresolvable random tissue in homogeneities in Radar imaging and ultrasound imaging from objects in undergo constructive and destructive interferences resulting in mottled b-scan image [5].

\subsection{Need for filtering}

Speckle [1] degrades the quality of Synthetic Aperture Radar and ultrasound images and thereby reducing the ability of a human observer to discriminate the fine details of investigative examination. Images with speckle noise will results in reducing the contrast of image and difficult to perform image processing operations like edge detection, segmentation. 


\subsection{Model of Speckle Noise}

\section{Noise Models}

An inherent characteristic of SAR and ultrasound images is the presence of speckle noise. Speckle noise is a random and deterministic in an image. Speckle has negative impact on SAR ultrasound images. Radical reduction in contrast resolution may be responsible for the poor effective resolution. In case of medical literatures, speckle noise is also known as texture. Generalized model of the speckle [3] is represented as,

$$
\mathrm{g}(\mathrm{n}, \mathrm{m})=\mathrm{f}(\mathrm{n}, \mathrm{m}) * \mathrm{u}(\mathrm{n}, \mathrm{m})+\xi(\mathrm{n}, \mathrm{m})
$$

Where $\mathrm{g}(\mathrm{n}, \mathrm{m})$, is the observed image, $\mathrm{u}(\mathrm{n}, \mathrm{m})$ is the multiplicative component, $\xi(\mathrm{n}, \mathrm{m})$ is the additive component of the speckle noise. Here $\mathrm{n}$ and $\mathrm{m}$ denotes the axial and lateral indices of the image samples.

\subsection{Noise in Synthetic Apertures Radar (SAR) Images}

Synthetic Apertures Radar (SAR) technique is popular because of its usability under various weather conditions, its ability to penetrate through clouds and soil [2]. A SAR image is a mean intensity estimate of the radar reflectivity of the region which is being imaged. Speckle noise in such system is to be referred as the difference between a measurement and the true mean value. Degraded image with speckle noise in ultrasound imaging is given by the equation;

$$
\mathrm{D}(\mathrm{X}, \mathrm{Y})=\mathrm{I}(\mathrm{X}, \mathrm{Y}) * \mathrm{~S}(\mathrm{X}, \mathrm{Y})
$$

Where, $\mathrm{D}(\mathrm{X}, \mathrm{Y})$ is the degraded ultrasound image with speckle, $\mathrm{I}(\mathrm{X}, \mathrm{Y})$ is the original image and $\mathrm{S}(\mathrm{X}, \mathrm{Y})$ is the speckle noise; where $(\mathrm{X}, \mathrm{Y})$ denotes the pixel location. The multiplicative nature of speckle complicates the noise reduction process [2].

\subsection{Noise Reduction in SAR Images}

\section{Speckle Noise Reduction And Enhancement Of SAR Images}

Speckle noise reduction in SAR images has been done using described algorithm below. An algorithm which use sampling to introduced the Discontinuity Adaptive Prior and Moment Estimation [2] within the ISUKF framework for speckle noise reduction. The stepwise algorithm is as given in Fig. 1.

Different filters give different statistical values for different images. Some filter will give best results for particular type of images like SRAD gives good results for ultrasonic images and AD [4], [6] gives good results for synthetic images. Hence the proposed algorithm automatically take decision regarding which filter is to be used for an image type since this algorithm selects the best output among the different filter outputs and displays the optimal results among the different results produced by different filtering techniques.

\subsection{Algorithm for Speckle Noise Reduction in SAR Images}

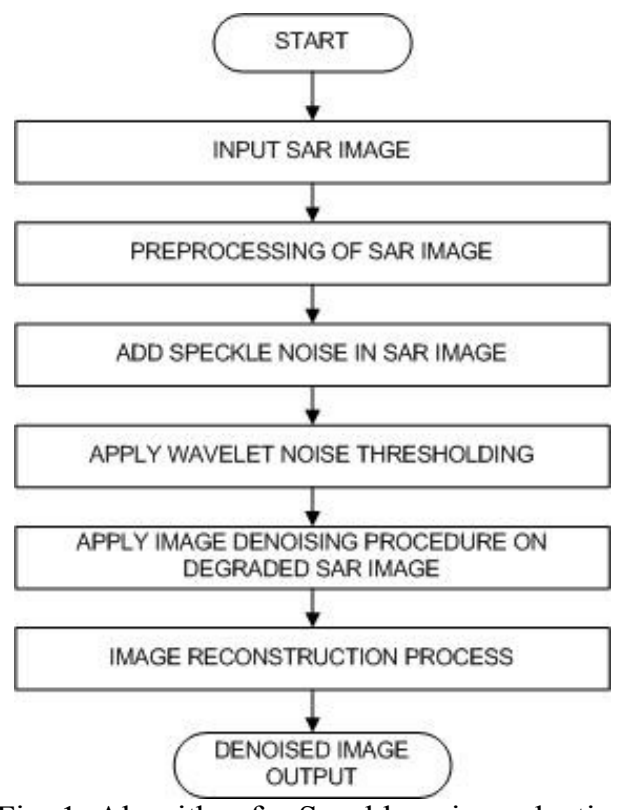

Fig. 1: Algorithm for Speckle noise reduction 


\section{Estimation Of Statistical Parameters}

The parameters which are used in the filter performance evaluation are Signal to Noise Ratio (SNR), Root Mean Square Error (RMSE), Peak Signal to Noise Ratio (PSNR) and Correlation Coefficient (COC),

\subsection{Estimation of Signal to Noise Ratio (SNR)}

SNR compares the level of desired signal to the level of background noise. The higher the SNR ratio, the lesser obtrusive the background noise is. SNR [7] in decibels is defined as

$$
\mathrm{SNR}=10 \log \left(\sigma_{\mathrm{g}}^{2} / \sigma_{\mathrm{e}}^{2}\right)
$$

Where, $\sigma_{\mathrm{g}}^{2}$, is the variance of the noise free image and $\sigma_{\mathrm{e}}^{2}$ is the variance of error (between the original and denoised image). Brighter regions have a stronger signal due to more light, resulting in higher overall SNR.

\subsection{Estimation of RMSE}

Mean square error (MSE) is given by

$$
\mathrm{MSE}=\Sigma^{\mathrm{N}}{ }_{\mathrm{i}=\mathrm{j}=1} \quad[\mathrm{f}(i, j)-F(i, j)]^{2} / N^{2}
$$

Where, $\mathrm{f}$ is the original image $\mathrm{F}$ is the image denoised with some filter and $\mathrm{N}$ is the size of image [8].

$$
\text { RMSE }=\sqrt{ } M S E
$$

\subsection{Estimation of PSNR} the image [8].

PSNR gives the ratio between possible power of a signal and the power of corrupting noise present in

$$
\text { PSNR }=20 \log 10(255 / \text { RMSE })
$$

Higher the PSNR gives lower the noise in the image i.e. higher image quality.

\subsection{Estimation of $\mathrm{COC}$}

Correlation gives the linear relationship between two signals with respect to strength and direction, and its value lies between -1 to +1 . The correlation is 1 for increasing linear relationship, -1 for decreasing linear relationship, for all the other cases the value lies between -1 to +1 . The closer the coefficient is to either -1 or +1 , the stronger the correlation between the signals [8].

$$
\mathrm{CoC}=\frac{\Sigma(\mathrm{g}-\dot{\mathrm{g}})(\hat{\mathrm{g}}-\dot{\mathrm{g}})}{\sqrt{\Sigma(\mathrm{g}-\dot{\mathrm{g}})^{2} \Sigma(\mathrm{g}-\dot{\mathrm{g}})^{2}}}
$$

Where, $g$ and $\hat{g}$ are original and images denoised with some filter respectively and $\dot{g}$ and $\hat{g}$ are the mean's of the original image and image denoised with a few filter respectively.

\section{Experimental Results}

The performance of the method that has been proposed is investigated with various simulations. Denoising is carried out for SAR image with Speckle noise of variance $\sigma 2=0.5,0.6,0.7$, using standard speckle filters and introduced filter. The performance of the various denoising methods is compared in Tables 1, 2 and 3 . We have presented a comparative study of various wavelet filters and standard speckle filters for SAR image in terms of PSNR (see Fig. 2 to Fig. 7). The performance of Speckle filters such as Kaun filter, Frost filter, the conventional approach in speckle filtering the homomorphic Wiener filter are measured here. We apply Matlab's spatially adaptive Wiener filter.

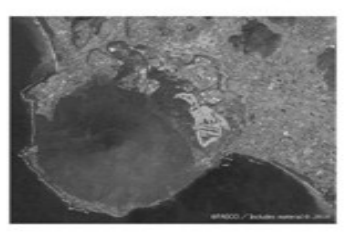

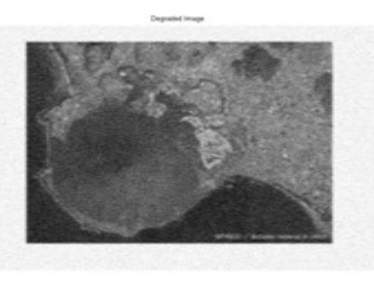

(b)

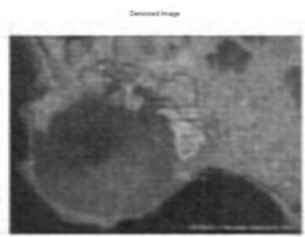

(c)

Fig.2: (a) Original SAR Image; (b) Degraded SAR Image by Speckle noise with variance 0.5; (c) Denoised SAR Image. 


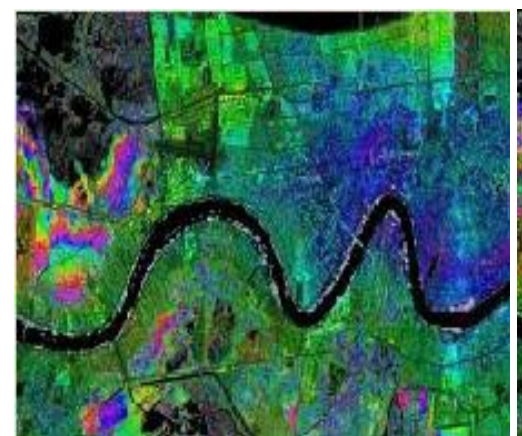

(a)

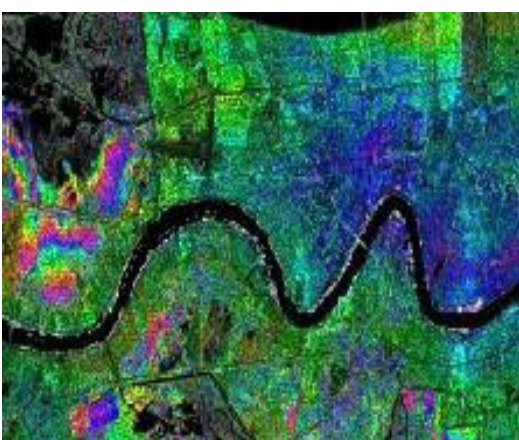

(b)

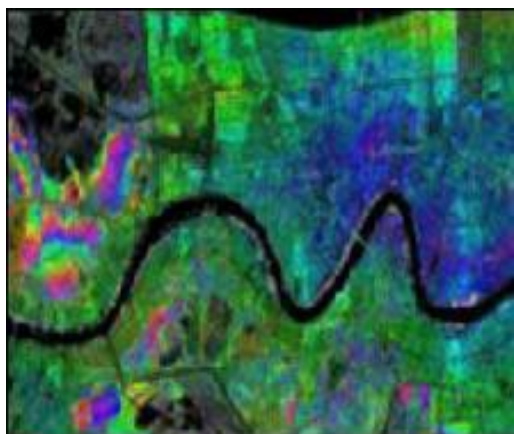

(c)

Fig.3: (a) Original SAR Image; (b) Degraded SAR Image by Speckle noise with variance 0.6; (c) Denoised SAR Image.

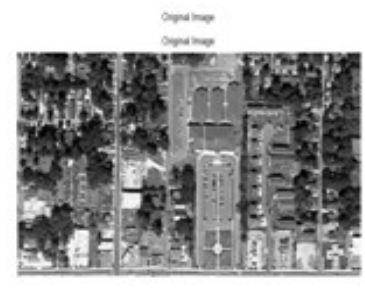

(a)

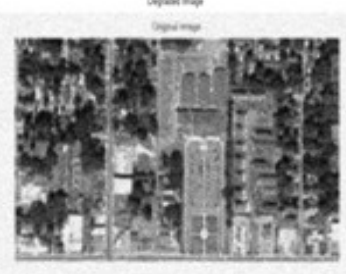

(b)

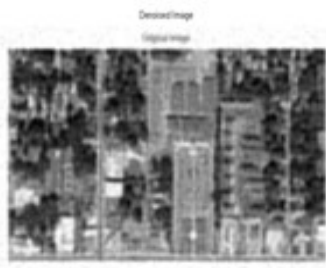

(c)

Fig.4: (a) Original SAR Image; (b) Degraded SAR Image by Speckle noise with variance 0.7; (c) Denoised SAR Image.

Table I Comparison of SNR, RMSE, PSNR and CoC of different De-noising Filters for SAR Images Corrupted by Speckle Noise with Variance $=0.5$ (Fig. 2)

\begin{tabular}{|l|l|l|l|l|}
\hline FILTER & SNR & RMSE & PSNR & CoC \\
\hline Original & 25.1435 & 8.1653 & 29.4228 & 0.9403 \\
\hline Lee & 27.9845 & 7.7612 & 31.2585 & 0.9783 \\
\hline Kuan & 30.1732 & 6.9342 & 31.4562 & 0.9761 \\
\hline Frost & 33.8652 & 7.0842 & 31.2790 & 0.9521 \\
\hline Wiener & 34.7631 & 5.8712 & 33.2894 & 0.9824 \\
\hline Proposed & 31.7612 & 6.4783 & 31.8723 & 0.9795 \\
\hline
\end{tabular}

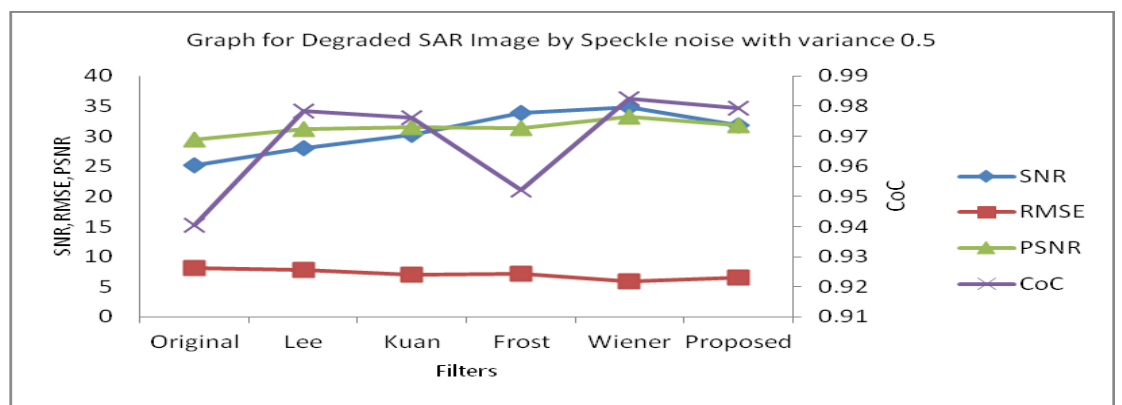

Fig. 5: Graph for Degraded SAR Image by Speckle noise with variance 0.5

Table II Comparison of SNR, RMSE, PSNR and CoC of different De-noising Filters for SAR Images Corrupted by Speckle Noise with Variance $=0.6$ (Fig. 3)

\begin{tabular}{|l|c|l|l|l|}
\hline FILTER & SNR & RMSE & PSNR & CoC \\
\hline Original & 23.4329 & 8.9543 & 27.4521 & 0.9125 \\
\hline Lee & 29.5783 & 7.1253 & 29.2152 & 0.9421 \\
\hline Kuan & 28.3463 & 6.8752 & 30.1353 & 0.9132 \\
\hline Frost & 31.4332 & 6.6316 & 30.8654 & 0.9257 \\
\hline Wiener & 32.2326 & 5.7432 & 32.8454 & 0.9733 \\
\hline Proposed & 31.3421 & 6.7533 & 31.2543 & 0.9722 \\
\hline
\end{tabular}




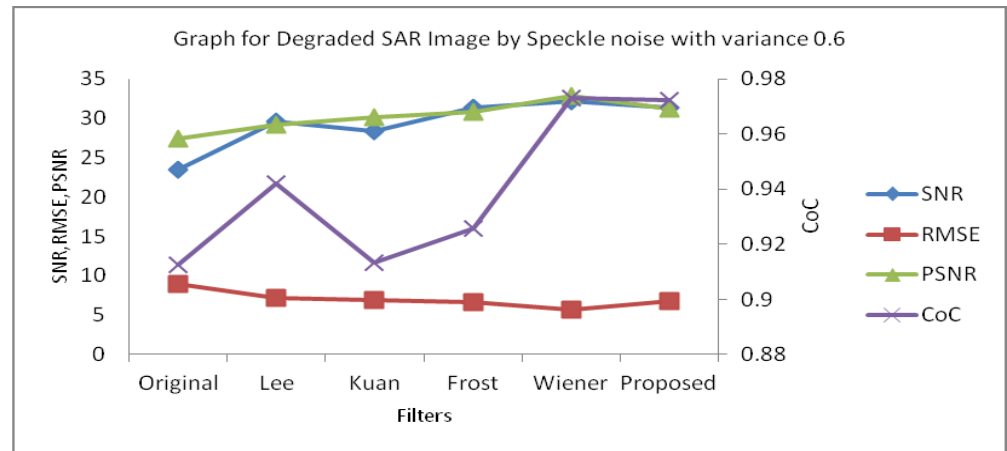

Fig. 6: Graph for Degraded SAR Image by Speckle noise with variance 0.6

Table III Comparison of SNR, RMSE, PSNR and CoC of different De-noising Filters for SAR Images Corrupted by Speckle Noise with Variance $=0.7$ (Fig. 4)

\begin{tabular}{|l|l|l|l|l|}
\hline FILTER & SNR & RMSE & PSNR & CoC \\
\hline Original & 22.4532 & 9.2352 & 26.7433 & 0.9002 \\
\hline Lee & 25.7325 & 8.3425 & 28.5313 & 0.9215 \\
\hline Kuan & 29.3422 & 7.4262 & 30.3152 & 0.9001 \\
\hline Frost & 34.4226 & 7.2315 & 30.1241 & 0.9387 \\
\hline Wiener & 31.4532 & 6.8532 & 31.9654 & 0.9742 \\
\hline Proposed & 31.2241 & 6.9311 & 30.8563 & 0.9711 \\
\hline
\end{tabular}

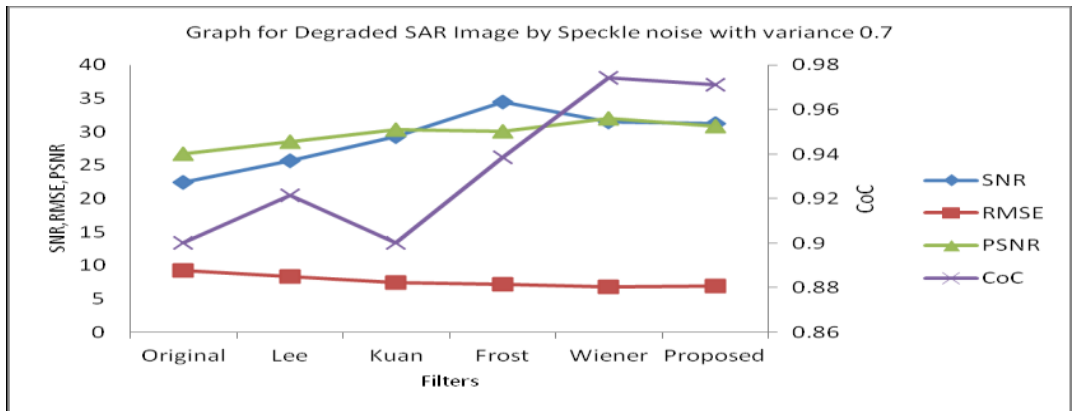

Fig. 7: Graph for Degraded SAR Image by Speckle noise with variance 0.7

\section{Conclusion}

We introduced a Speckle noise reduction model in Synthetic Aperture Radar (SAR) imagery. The model preserve the appearances of structured regions. In case of Synthetic Aperture Radar (SAR) Images, Texture and land surfaces have been enhanced. The performance of the algorithm has been tested using visual performance measures. Many of the methods are failure to remove speckle noise present in the Synthetic Aperture Radar (SAR) images, since the information about the variance of the noise may not be able to identify by the methods. Introduced model automatically collect the information about the noise variance. Performance of the Speckle noise reduction model for Synthetic Aperture Radar (SAR) imagery is well as compared to other filters. The results shows very closed equivalency in between SAR original images and SAR denoised i.e. enhanced images.

\section{References}

[1] J. W. Goodman, "Some fundamental properties of speckle," J. Opt. Soc. Amer., vol. 66, no. 11, pp. 1145-1149, 1976.

[2] M. P.Wachowiak, R. Smolikova, J. M. Zurada, and A. S. Elmaghraby, "Estimation of K-distribution parameters using neural networks," IEEE Trans. Biomed. Eng., vol. 49, no. 6, pp. 617-620, Jun. 2002.

[3] J. M. Park, W. J. Song, and W. A. Pearlman, "Speckle filtering of sar images based on adaptive windowing," Proc. Inst. Elect. Eng. Vis. Image Signal Process., vol. 146, no. 4, 1999.

[4] Yongjian Yu, Janelle A. Molloy and Scott T. Acton "Generalized Speckle Reducing Anisotropic Diffusion for Ultrasound Imagery", 17th IEEE Symposium on Computer-Based Medical Systems, 2004, pages: 279- 284

[5] C. B. Burckhardt, "Speckle in ultrasound b-mode scans," IEEE Trans. Sonics Ultrason., vol. SU-25, no. 1, pp. 1-6, 1978.

[6] Y. Yu and S. T. Acton, "Speckle reducing anisotropic diffusion," IEEE Trans. Image Process., vol. 11, no. 11, pp. 1260-1270, Nov. 2002 .

[7] Scott T. Acton, "De-convolution speckle reducing anisotropic diffusion", IEEE International Conference on Image Processing, 2005, Vol.1, pages: I- 5-8

[8] A. Achim, E.E. Kuruoglu and J. Zerubia, "SAR image filtering based on the heavy - tailed Rayleigh model", IEEE Trans. Image Process., Vol. 1, No. 9, pp. 2686-2693, Sept. 2006. 


\section{Biography}

K. M. Sharavana Raju, Lecturer in Department of Computer Science, College of Computer Science and Information Systems, Jazan University, Jazan, Kingdom of Saudi Arabia. He received his M.Sc., (Physics), PGDCA and M.C.A., degrees from Bharathidasan University, Tiruchirapalli, Tamilnadu, India and M.Phil.,(CS) Degree from Periyar University, Salem, Tamilnadu, India. Currently he is pursuing Ph.D., in Computer Science at Bharathiar University, Coimbatore, Tamilnadu, India. He has published papers in 5 International journals, several National journals and also attended 3 International conferences and several National conferences and workshops. He is having 19 years of collegiate teaching experience, which includes 1 year at Libya. His areas of research include Digital Image processing on Satellite Images and Segmentation methods.

Mohammad Shahnawaz Nasir, Lecturer in College of Computer Science and Information System, Jazan University, Jazan, Kingdom of Saudi Arabia. He received his Master in Computer Science \& Applications (MCA) and Master in Science (M.Sc.) Physics, Electronic Specializations Degrees from Aligarh Muslim University, India. Previously he worked as faculty with AOU (KSA), JMI (New-Delhi) and AMU (Aligarh, India). His work experience in Saudi Arabia also includes Web, Network, and Database Administration. Currently he is pursuing Ph.D., in Computer Science at Magadh University, Bodh-Gaya, India. His research areas are Data Mining, Biometrics and Image Processing.

Dr. T. Meera Devi, Associate Professor, in the Department of Electronics and Communication Engineering, Kongu Engineering College, Perundurai, Tamilnadu, India. She received her Bachelor's degree in Electronics and Communication Engineering, in 1996 at The Indian Engineering College, Vadakkankulam, Tamilnadu, India; Master's degree in Applied Electronics in 2005 and Ph.D ., in Signal Processing from Anna University, Chennai, Tamilnadu, India in 2013. She has been in the teaching profession for the past fourteen years. Her areas of academic interest include Soft Computing, Signal Processing and Image Processing. 